\title{
Adaptive Guaranteed-Performance Consensus Control for Multi-Agent Systems with an Adjustable Convergence Speed
}

\author{
Haiying Ma, ${ }^{1}$ Xiao Jia, ${ }^{2}$ Ning Cai $(\mathbb{D})^{3}$ and Jianxiang Xi ${ }^{2}{ }^{2}$ \\ ${ }^{1}$ School of Economics, Northwest Minzu University, Lanzhou 730030, China \\ ${ }^{2}$ Rocket Force University of Engineering, Xian 710025, China \\ ${ }^{3}$ School of Automation, Beijing University of Posts and Telecommunications, Beijing 100876, China \\ Correspondence should be addressed to Jianxiang Xi; xijx07@mails.tsinghua.edu.cn
}

Received 31 January 2019; Revised 31 May 2019; Accepted 10 June 2019; Published 2 July 2019

Academic Editor: Zhengqiu Zhang

Copyright (C) 2019 Haiying Ma et al. This is an open access article distributed under the Creative Commons Attribution License, which permits unrestricted use, distribution, and reproduction in any medium, provided the original work is properly cited.

\begin{abstract}
In this paper, adaptive guaranteed-performance consensus control problems for multiagent systems with an adjustable convergence speed are investigated. A novel adaptive guaranteed-performance consensus protocol is proposed, where the communication weights can be adaptively regulated. By the state space decomposition method and the stability theory, sufficient conditions for guaranteed-performance consensus are obtained and the guaranteed-performance cost is determined. Moreover, the lower bound of the convergence coefficient for multiagent systems is deduced, which is linearly adjustable approximately by changing the adaptive control gain. Finally, simulation examples are introduced to demonstrate theoretical results.
\end{abstract}

\section{Introduction}

In recent years, by the incentive effects of spacious applications, such as synchronization $[1,2]$, formation control [3-5], cluster flight [6-8], and other fields [9-11], there is considerable attention in distributed cooperative control of multiagent systems. As a significant topic in cooperative control, consensus, which means that all agents in a multiagent system achieve an agreement on some factors by designing reasonable consensus control protocols, arouses extensive interest from investigators, and some meaningful relevant works have been developed in [12-15], where the consensus performance is not taken into account.

In some practical complex multiagent systems, except for the requirements of achieving consensus, the consensus performance also needs to be taken into consideration. There is a representative example in [16]. It is shown that when a particular task is carried out by multiple mobile vehicles, the distance performance is likely to be a critical factor due to restricted resource. Generally speaking, when consensus should be achieved in multiagent systems and under the condition that certain cost functions included in constraints are identified as minimum or maximum, one can model these correlative issues as optimal or suboptimal consensus. It should be pointed out that researchers always use guaranteedperformance consensualization to realize optimal consensus control. In order to deal with the optimal consensus problem for first-order multiagent systems with both continuous-time and discrete-time dynamics, LQR-based optimal algorithms were proposed and the optimal Laplacian matrices were deduced in [17]. Guan et al. [18] studied the guaranteedperformance consensus problem for second-order multiagent systems, and a performance function was provided for evaluating the performance of each agent based on impulsive control methods. Furthermore, for high-order multiagent systems, some researchers also introduced effective guaranteed-performance consensus algorithms to solve existing problems in [19-22]. Although many innovative and significant results were given in [16-22], the convergence speed of multiagent systems was not considered, which was one of the most important aspects for the system evaluation.

As is well known, the consensus speed was firstly referred by Olfati-Saber in $[23,24]$. Meanwhile, the relationship between the consensus speed and the algebraic connectivity of the system topology was presented, which was given the formal definition in [25]. It is no doubt that, in view of the 
algebraic connectivity, many researchers have made much effort on improving the convergence speed by the transformation or reconstruction of topology structures shown in [26-30]. Ultra-fast consensus was achieved in small-world networks transformed from regular networks in [26]. An iterative algorithm employing semidefinite programming was applied in [27]. Moreover, based on hierarchical structures mentioned in [28, 29], a multilayer hierarchical structure algorithm was proposed in [30-32], which made it available to adjust the convergence speed. However, in practice, the system topology is always determined. In this case, optimizing consensus control protocols is a more useful way to change the algebraic connectivity with given topology structures. Yuan et al. [33] used partial information of second-order neighbors in different cases to accelerate distributed consensus through choosing suitable edges, and a similar approach was applied in [34]. In order to accelerate the convergence speed and reduce the communication cost among nodes with undirected topologies, pseudo multihop relay algorithms were proposed in [35], which was also extended to highorder multiagent systems with directed topologies in [36]. In addition, predictive control methods were introduced to make multiagent systems achieve fast consensus in $[37,38]$. It should be pointed out that the above interesting results did not consider the guaranteed-performance condition.

In some practical applications, such as movement and communication, each agent in multiagent systems may have limited energy supply. However, a lot of research works about the consensus control for multiagent systems do not take the above-mentioned important factor into consideration. This paper focuses on the guaranteed-performance consensus analysis of multiagent systems, which can deal with the limited energy problem effectively. Furthermore, the convergence speed plays an essential role in the consensus performance of multiagent systems. Although the convergence speed can be adjusted by changing the algebraic connectivity related to the topology structure, it is usually difficult to change the topology structure in practice. This paper finds that the adjustable convergence speed can be obtained by changing the adaptive control gain, which owns great engineering significance.

The current paper studies adaptive guaranteed-performance consensus control for multiagent systems with an adjustable convergence speed. As far as I am concerned, the contribution of this paper is the following threefold: (i) A new adaptive guaranteed-performance consensus protocol for first-order multiagent systems is proposed, and the communication weights among nodes in the system topology can be adaptively adjustable by state errors between each agent and its neighbors. (ii) The multiagent system is decomposed into two subsystems which determine the consensus motion and the disagreement motion, respectively. Sufficient conditions for guaranteed-performance consensus are obtained, and the guaranteed-performance cost is determined at the same time. (iii) The convergence coefficient is defined for multiagent systems under an adaptive consensus protocol, and the lower bound of the convergence coefficient is determined, which is related to the adaptive control gain and the minimum nonzero eigenvalue.
Compared with the existing relevant works about the consensus problem of multiagent systems, this paper has following novelties. Firstly, a novel consensus control strategy is proposed to guarantee the consensus performance of multiagent systems. Note that it corresponds with practical applications, which means that many systems in aviation and aerospace are constrained by limited energy. However, this method is not available in [12-15]. Secondly, the communication weights are regulated by both state errors and the adaptive control gain, while the communication weights cannot be regulated in [12-15]. Thirdly, in view of the given system topology and the huge communication burden, by changing the adaptive control gain rather than the algebraic connectivity related to the topology network, the convergence speed can be adjusted in this paper, but the adjustable convergence speed is not mentioned in [12-15].

The remainder of the paper is organized as follows. Section 2 states some useful materials and important methods to deal with the guaranteed-performance consensus of multi-agent systems and the convergence coefficient associated with the convergence speed. The theoretical results are illustrated via simulation examples in Section 3. Finally, Section 4 gives some discussion and draws the conclusion.

Notations. $R^{N}$ is the real column vector space of dimension $N$, and $R^{N \times N}$ denotes the set of $N \times N$ dimensional real matrices. The transpose of a matrix $Q$ is denoted as $Q^{T} . \boldsymbol{I}_{M}$ stands for the identity matrix with dimension $M .0$ represents the zero column vector with appropriate dimension. Let $\mathbf{1}_{M}=$ $[1, \cdots, 1]^{T}$ be the vector of $M$ ones with all components 1 .

\section{Materials and Methods}

2.1. Preliminaries and Problem Description. This section mainly introduces some basic concepts of the graph theory and presents the problem description.

(A) Algebraic Graph Theory. A connected undirected graph $G=(V, E, W)$ can be used to describe the information interchange among agents in a multiagent system, where $V=$ $\left\{v_{1}, v_{2}, \cdots, v_{N}\right\}, E=V \times V$ and $W=\left[w_{i j}\right] \in \mathrm{R}^{N \times N}$ stand for a set of vertices, a set of edges, and the adjacency matrix related to the graph $G$, respectively. $e_{i j}=\left(v_{i}, v_{j}\right) \in E$ represents an edge in $G$, where the information exchange exists between node $v_{i}$ and node $v_{j}$, and node $v_{i}$ is called a neighbor of node $v_{j}$. Note that we can describe all neighbors of node $v_{j}$ as an index set $N_{j}=\left\{i \mid\left(v_{i}, v_{j}\right) \in E\right\}$. For the undirected graph $G,\left(v_{i}, v_{j}\right) \Longleftrightarrow\left(v_{j}, v_{i}\right)$. The adjacency element $w_{i j}$ corresponds to the interaction strength between node $v_{i}$ and node $v_{j}$, where $w_{i j}=1$ shows $e_{i j}=\left(v_{i}, v_{j}\right) \in E$ and $w_{i j}=0$ shows $e_{i j}=\left(v_{i}, v_{j}\right) \notin E$. It is assumed that there is no self-edge for each node; that is, $w_{i i}=0$. Define by $L=\left[l_{i j}\right] \in R^{N \times N}$ the Laplacian matrix of $G$, where $l_{i i}=\sum_{j=1, j \neq i}^{N} w_{i j}$ and $l_{i j}=-w_{i j}(i \neq j)$. According to the definition of connected undirected graphs, one eigenvalue of $L$ is zero, and the remaining eigenvalues are positive.

Remark 1. From the graph theory, one can see that it is meaningful to describe the information interchange of 
multiagent systems by connected undirected graph $G$ shown in Simulation Results. The reason is that each agent can be represented by a node of this graph. In this case, the adjacency matrix of $G$ is available in the adaptive guaranteedperformance consensus control protocol. More importantly, the Laplacian matrix of $G$ plays a significant role in the adaptive guaranteed-performance consensus analysis and even make a difference on the theoretical results. Compared with the application of the graph theory in [39], one can obtain that the research objectives are different. The graph theory in this paper shows the information interchange of multiagent systems, but the graph theory in [zhang1] is used for complex-valued neural networks. In this case, a node of graphs in this paper stands for an agent of multiagent systems, whose information transmission is bidirectional. However, a node of graphs in [39] represents a neuron of neural networks, whose information transmission is certainly unidirectional.

(B) Problem Description. Consider a complex multiagent system with $M$ nodes with each node modeled as

$$
\dot{x}_{k}(t)=u_{k}(t) \quad(k=1,2, \cdots, M),
$$

where $x_{k}(t) \in \mathbf{R}$ is the state of the $k$ th node to be coordinated and $u_{k}(t) \in \mathbf{R}$ is the control input of the $k$ th node to be designed according to the information of its neighboring nodes. The system topology among nodes is modeled as a connected undirected graph.

The following adaptive guaranteed-performance control protocol is proposed:

$$
\begin{aligned}
u_{k}(t) & =\alpha \sum_{j \in N_{k}} \beta_{k j} w_{k j}(t)\left(x_{j}(t)-x_{k}(t)\right), \\
\dot{w}_{k j}(t) & =\alpha\left(x_{j}(t)-x_{k}(t)\right)^{2}, \\
J_{r} & =\frac{1}{M} \sum_{k=1}^{M} \sum_{j=1}^{M} \int_{0}^{+\infty} \zeta\left(x_{j}(t)-x_{k}(t)\right)^{2} \mathrm{~d} t,
\end{aligned}
$$

where $\alpha$ is the adaptive control gain, $\zeta$ represents the performance coefficient with $\zeta>0, N_{k}$ denotes the neighbor set of node $k, \beta_{k j}$ stands for the connection state of the edge between node $j$ and node $k$, which equals one if node $j$ is connected to node $k$ and identically equals to zero otherwise, and $w_{k j}(t)$ and $\dot{w}_{k j}(t)$ are the adaptively regulated weight of the edge from node $j$ to node $k$ and its time derivative, respectively.

It can be found that if $\alpha>0, w_{k j}(t)$ is nondecreasing time-varying and its variation is closely associated with the state errors between two agents. Furthermore, the growth rate of $w_{k j}(t)$ is faster when the state errors are greater, which means that the greater effect is given to adjust the state errors between them. Especially, $w_{k j}(t)$ is not changed when the states of two agents are equal. Without loss of generality, it is assumed that the initial value of weight $w_{k j}(0)$ is 1 and the upper bound of $w_{k j}(t)$ is $w_{k j m}$.

In view the undirected topology $G$, one can easily see that nodes in the topology correspond to agents of multiagent systems. In this case, let $\boldsymbol{x}(t)=\left[x_{1}(t), x_{2}(t), \cdots, x_{M}(t)\right]^{T}$, then a compact form of system (1) with control protocol (2) is produced as follows:

$$
\dot{\boldsymbol{x}}(t)=-\alpha L_{w(t)} \boldsymbol{x}(t),
$$

where $L_{w(t)}$ stands for the Laplacian matrix of $G$.

The definition of the adaptive guaranteed-performance consensus for multiagent system (1) is described as follows.

Definition 2. Combined with control protocol (2), multiagent system (1) is said to achieve adaptive guaranteedperformance consensus if there exists $\alpha$ such that $\lim _{t \rightarrow \infty}\left(x_{k}(t)-x_{j}(t)\right)=0(k, j=1,2, \cdots, M)$, and $J_{r} \leq J_{r}^{*}$ in view of any bounded initial states $x_{k}(0)(k=1,2, \cdots, M)$, where $J_{r}^{*}$ is known as the guaranteed-performance cost.

Remark 3. The aim of this paper is to obtain a suitable adaptive control gain and the guaranteed-performance cost, so that multiagent system (1) can achieve adaptive guaranteed-performance consensus. Moreover, there are two main features in adaptive guaranteed-performance control protocol (2). Firstly, the guaranteed-performance function $J_{r}$ associated with the performance coefficient and state errors is proposed. In this case, the consensus performance of multiagent systems is guaranteed, which is more meaningful compared with [12-15]. Secondly, the adaptive control gain is designed, and it can effectively regulate the time-varying communication weights, which plays an important role in the consensus analysis for multiagent systems. However, this factor is not referred in [12-15].

Remark 4. There is no doubt that the research method between the consensus problem of multiagent systems and the synchronization problem of complex networks is the same, which is sufficiently introduced in [40]. In order to transfer consensus problems of multiagent systems into stability problems of several matrices with the same lower dimension, a novel unified framework was presented, which is essentially coincident with the main stability function method used in the synchronization problem of complex networks. Thus, the integral inequality techniques can also be applied in the consensus control analysis, which was used to present two new sufficient conditions on global asymptotic synchronization for the drive-response inertial delayed neural networks under two new controllers in [41].

2.2. Main Results. In the following theoretical analysis, sufficient conditions for multiagent system (1) to achieve adaptive guaranteed-performance consensus are obtained by designing the adaptive control gain $\alpha$, then the guaranteedperformance cost $J_{r}^{*}$ is determined. Moreover, the consensus convergence speed is discussed, and the lower bound of the convergence coefficient is presented to indicate the convergence speed. We prove that it is an effective way to linearly regulate the consensus convergence speed by changing $\alpha$.

(C) Adaptive Guaranteed-Performance Consensus Analysis. In the first place, the state space decomposition approach is proposed to transform the dynamics of multiagent system 
(1). Denote by $\mathbf{0}=\lambda_{1}<\lambda_{2} \leq \cdots \leq \lambda_{M}$ the eigenvalues of $L_{w(0)}$. Then according to matrix theory, one can find that there exists an orthogonal matrix $Q=\left[\mathbf{1}_{M} / \sqrt{M} \widetilde{Q}\right]$ with $\mathbf{1}_{M}=[1,1, \cdots, 1]^{T} \in \mathbf{R}^{M}$ satisfying

$$
Q^{T} L_{w(0)} Q=\left[\begin{array}{cc}
0 & \mathbf{0}^{T} \\
\mathbf{0} & \Omega_{(0)}
\end{array}\right],
$$

where $\Omega_{(0)}=\widetilde{Q}^{T} L_{w(0)} \widetilde{Q}=\operatorname{diag}\left\{\lambda_{2}, \lambda_{3}, \cdots, \lambda_{M}\right\}$. Let

$$
\tilde{\boldsymbol{x}}(t)=Q^{T} \boldsymbol{x}(t)=\left[\tilde{x}_{1}^{T}(t), \boldsymbol{\mu}^{T}(t)\right]^{T},
$$

where $\boldsymbol{\mu}(t)=\left[\widetilde{x}_{2}^{T}(t), \widetilde{x}_{3}^{T}(t), \cdots, \widetilde{x}_{M}^{T}(t)\right]^{T}$, then one can get that multiagent system (3) can be rewritten as

$$
\begin{aligned}
& \dot{\tilde{x}}_{1}(t)=0, \\
& \dot{\boldsymbol{\mu}}(t)=-\alpha \widetilde{\mathrm{Q}}^{T} L_{w(t)} \widetilde{\mathrm{Q}} \boldsymbol{\mu}(t) .
\end{aligned}
$$

Let $\boldsymbol{e}_{i}(i=1,2, \cdots, M)$ denote $M$-dimensional unit vectors with the $i$ th element 1 and 0 elsewhere. Define

$$
\begin{aligned}
& \boldsymbol{x}_{c}(t):=Q \boldsymbol{e}_{1} \widetilde{x}_{1}(t)=\frac{1}{\sqrt{M}} \widetilde{x}_{1}(t), \\
& \boldsymbol{x}_{\bar{c}}(t):=\sum_{i=2}^{M} Q \boldsymbol{e}_{i} \otimes \tilde{x}_{i}(t) .
\end{aligned}
$$

Due to

$$
\sum_{i=2}^{M} \boldsymbol{e}_{i} \otimes \tilde{x}_{i}(t)=\left[\mathbf{0}, \boldsymbol{\mu}^{T}(t)\right]^{T}
$$

one can obtain from (8) that

$$
\boldsymbol{x}_{c}(t)=Q\left[\widetilde{x}_{1}^{T}(t), \mathbf{0}\right]^{T} .
$$

Similar to the above analysis, it follows from (9) that

$$
\boldsymbol{x}_{\bar{c}}(t)=Q\left[0, \boldsymbol{\mu}^{T}(t)\right]^{T} .
$$

According to the definition of orthogonal matrices, one can see that $Q$ is nonsingular. Thus it holds that $\boldsymbol{x}_{c}(t)$ and $\boldsymbol{x}_{\bar{c}}(t)$ are linearly independent with each other by (11) and (12). Due to $\widetilde{\boldsymbol{x}}(t)=Q^{T} \boldsymbol{x}(t)=\left[\widetilde{x}_{1}^{T}(t), \boldsymbol{\mu}^{T}(t)\right]^{T}$, it can be shown that

$$
\boldsymbol{x}(t)=\boldsymbol{x}_{c}(t)+\boldsymbol{x}_{\bar{c}}(t) .
$$

From the form of $\boldsymbol{x}_{c}(t)$ in (8), one can show that multiagent system (1) under control protocol (2) can be adaptively guaranteed-performance consensualizable if and only if $\lim _{t \rightarrow \infty} \boldsymbol{\mu}(t)=0$; in other words, subsystems (6) and (7) are presented respectively to indicate the consensus motion and relative state motion of multiagent system (1).

In the following theorem, sufficient conditions for adaptive guaranteed-performance consensus are obtained, which means that distributed guaranteed-performance consensus design for multiagent system (1) is derived.
Theorem 5. Multiagent (1) with control protocol (2) achieves adaptive guaranteed-performance consensus if $\alpha \geq 2 \zeta$. In this case, the guaranteed-performance cost satisfies that

$$
\begin{aligned}
J_{r}^{*}= & \boldsymbol{x}^{T}(0)\left(\boldsymbol{I}_{M}-\frac{1}{M} \mathbf{1}_{M} \mathbf{1}_{M}^{T}\right) \boldsymbol{x}(0) \\
& +\alpha \int_{0}^{+\infty} \boldsymbol{x}^{T}(t)\left(\boldsymbol{I}_{M}-\frac{1}{M} \mathbf{1}_{M} \mathbf{1}_{M}^{T}\right) \boldsymbol{x}(t) \mathrm{d} t .
\end{aligned}
$$

Proof. To begin with, we prove $\lim _{t \rightarrow \infty} \mu(t)=\mathbf{0}$ under $\alpha \geq$ $2 \zeta>0$. Consider a Lyapunov function candidate as follows:

$$
\begin{aligned}
V(t)= & \boldsymbol{\mu}^{T}(t) \boldsymbol{\mu}(t)+\sum_{k=1}^{M} \sum_{j=1}^{M} \frac{\left(w_{k j}(t)-w_{k j}(0)\right)^{2}}{2} \\
& +\frac{1}{2 M} \sum_{k=1}^{M} \sum_{j=1}^{M}\left(w_{k j m}-w_{k j}(t)\right) .
\end{aligned}
$$

Because of $w_{k j m} \geq w_{k j}(t)$, one can get that $V(t) \geq 0$ in spite of the value of $\alpha$. Furthermore, taking the time derivative of $V(t)$ along the trajectory of subsystem (7), one has

$$
\begin{aligned}
\dot{V}(t) & =\left(-\alpha \boldsymbol{\mu}^{T}(t) \widetilde{\mathrm{Q}}^{T} L_{w(t)}^{T} \widetilde{\mathrm{Q}} \boldsymbol{\mu}(t)\right. \\
- & \left.\boldsymbol{\mu}^{T}(t) \alpha \widetilde{\mathrm{Q}}^{T} L_{w(t)} \widetilde{\mathrm{Q}} \boldsymbol{\mu}(t)\right) \\
+ & \sum_{k=1}^{M} \sum_{j=1}^{M}\left(w_{k j}(t)-w_{k j}(0)\right) \dot{w}_{k j}(t)-\frac{1}{2 M} \\
& \cdot \sum_{k=1}^{M} \sum_{j=1}^{M} \dot{w}_{k j}(t) .
\end{aligned}
$$

Due to the assumption of the undirected connected graph $G$, it can be seen that $L_{w(t)}^{T}=L_{w(t)}$. Then since equation $Q Q^{T}=$ $I_{M}$ holds, one can easily obtain $\widetilde{Q} \widetilde{Q}^{T}=L_{M}$, where $L_{M}$ is the Laplacian matrix of a complete graph with the weights of all edges $1 / M$. Moreover, one has

$$
\frac{1}{2} \sum_{k=1}^{M} \sum_{j=1}^{M} w_{k j}(t)\left(x_{j}(t)-x_{k}(t)\right)^{2}=\boldsymbol{x}(t)^{T} L_{w(t)} \boldsymbol{x}(t)
$$

Then by (2) and (17), it can be seen that

$$
\begin{aligned}
& \sum_{k=1}^{M} \sum_{j=1}^{M}\left(w_{k j}(t)-w_{k j}(0)\right) \dot{w}_{k j}(t) \\
& \quad=2 \alpha \boldsymbol{x}^{T}(t) L_{w(t)} \boldsymbol{x}(t)-2 \alpha \boldsymbol{x}^{T}(t) L_{w(0)} \boldsymbol{x}(t) .
\end{aligned}
$$

In addition, it can be also obtained that

$$
\frac{1}{2 M} \sum_{k=1}^{M} \sum_{j=1}^{M} \dot{w}_{k j}(t)=\alpha \boldsymbol{x}^{T}(t) L_{M} \boldsymbol{x}(t) .
$$


Substituting (18) and (19) into (16), it can be deduced that

$$
\begin{aligned}
\dot{V}(t)= & -2 \alpha \boldsymbol{\mu}^{T}(t) \widetilde{Q}^{T} L_{w(t)} \widetilde{Q} \boldsymbol{\mu}(t) \\
& +2 \alpha \boldsymbol{x}^{T}(t) L_{w(t)} \boldsymbol{x}(t)-2 \alpha \boldsymbol{x}^{T}(t) L_{w(0)} \boldsymbol{x}(t) \\
& -\alpha \boldsymbol{x}^{T}(t) L_{M} \boldsymbol{x}(t) \\
= & -2 \alpha \boldsymbol{\mu}^{T}(t) \widetilde{Q}^{T} L_{w(0)} \widetilde{Q} \boldsymbol{\mu}(t) \\
& -\alpha \boldsymbol{\mu}^{T}(t) \widetilde{Q}^{T} L_{M} \widetilde{Q} \boldsymbol{\mu}(t) \\
= & -\alpha \sum_{k=2}^{M} \widetilde{x}_{i}^{T}(t)\left(2 \lambda_{k}+1\right) \widetilde{x}_{k}(t) .
\end{aligned}
$$

Moreover, it should be pointed that $\lambda_{k}>0(k=2,3, \cdots, M)$, then if $\alpha>0$, the following inequality holds:

$$
\begin{aligned}
\dot{V}(t) & \leq-\alpha\left(2 \lambda_{2}+1\right) \sum_{k=2}^{M} \tilde{x}_{k}^{T}(t) \tilde{x}_{k}(t) \\
& =-\alpha\left(2 \lambda_{2}+1\right)\|\boldsymbol{\mu}(t)\|^{2} .
\end{aligned}
$$

Accordingly, $\boldsymbol{\mu}(t)$ converges to $\mathbf{0}$; that is, multiagent system (1) under control protocol (2) can be adaptively consensualizable.

In the following discussion, the guaranteed-performance cost is determined. Firstly, one can obtain from (16) that

$$
\begin{aligned}
\sum_{k=1}^{M} & \sum_{j=1}^{M}\left(x_{j}(t)-x_{k}(t)\right)^{T} \zeta\left(x_{j}(t)-x_{k}(t)\right) \\
& =2 M \zeta \boldsymbol{x}^{T}(t) L_{M} \boldsymbol{x}(t)=2 M \zeta \boldsymbol{\mu}^{\mathrm{T}}(t) \widetilde{Q}^{T} L_{M} \widetilde{Q} \boldsymbol{\mu}(t) \\
& =2 M \zeta \sum_{k=2}^{M} \tilde{x}_{k}^{T}(t) \tilde{x}_{k}(t) .
\end{aligned}
$$

Let $H>0$. Then define

$$
\begin{aligned}
J_{r}^{H} & \triangleq \frac{1}{M} \\
& \cdot \sum_{k=1}^{M} \sum_{j=1}^{M} \int_{0}^{H}\left(x_{j}(t)-x_{k}(t)\right)^{T} \zeta\left(x_{j}(t)-x_{k}(t)\right) \mathrm{d} t
\end{aligned}
$$

Substituting (22) into (23) leads to

$$
J_{r}^{H}=2 \zeta \int_{0}^{H} \sum_{k=2}^{M} \tilde{x}_{k}^{T}(t) \tilde{x}_{k}(t) \mathrm{d} t .
$$

Furthermore, it can be shown that

$$
\int_{0}^{H} \dot{V}(t) \mathrm{d} t-V(H)+V(0)=0 .
$$

Since $\lim _{t \rightarrow \infty}\left(w_{k j}(t)-w_{k j m}\right)=0$, one can easily get that

$$
\lim _{H \rightarrow \infty} \sum_{k=1}^{M} \sum_{j=1, k \neq j}^{M}\left(w_{k j}(H)-w_{k j m}\right)=0 .
$$

Note that $\dot{V}(t)=-\alpha \sum_{k=2}^{M} \tilde{x}_{k}^{T}(t)\left(2 \lambda_{k}+1\right) \tilde{x}_{k}(t) \leq$ $-\alpha \sum_{k=2}^{M} \tilde{x}_{k}^{T}(t) \tilde{x}_{k}(t)$. Then if $\alpha \geq 2 \zeta$, it follows from (24), (25), and (26) that

$$
\begin{aligned}
\lim _{H \rightarrow \infty} J_{r}^{H}= & \lim _{H \rightarrow \infty} 2 \zeta \int_{0}^{H} \sum_{k=2}^{M} \tilde{x}_{k}^{T}(t) \tilde{x}_{k}(t) \mathrm{d} t \\
\leq & \boldsymbol{\mu}^{T}(0) \boldsymbol{\mu}(0) \\
& +\frac{1}{2 M} \sum_{k=1}^{M} \sum_{j=1, k \neq j}^{M}\left(w_{k j m}-w_{k j}(0)\right) .
\end{aligned}
$$

Due to $\boldsymbol{\mu}(t)=\widetilde{Q}^{T} \boldsymbol{x}(t)$ and $\widetilde{Q} \widetilde{Q}^{T}=L_{M}$, one gets

$$
\boldsymbol{\mu}^{T}(0) \boldsymbol{\mu}(0)=\boldsymbol{x}^{T}(0)\left(\boldsymbol{I}_{M}-\frac{1}{M} \mathbf{1}_{M} \mathbf{1}_{M}^{T}\right) \boldsymbol{x}(0) .
$$

Since $\lim _{t \rightarrow \infty}\left(w_{k j}(t)-w_{k j m}\right)=0$, one has

$$
\begin{gathered}
\sum_{k=1}^{M} \sum_{j=1, k \neq j}^{M}\left(w_{k j m}-w_{k j}(0)\right)=\sum_{k=1}^{M} \sum_{j=1, k \neq j}^{M} \int_{0}^{+\infty} \dot{w}_{k j}(t) \mathrm{d} t \\
=2 M \alpha \int_{0}^{+\infty} \boldsymbol{x}^{T}(t)\left(\boldsymbol{I}_{M}-\frac{1}{M} \mathbf{1}_{M} \mathbf{1}_{M}^{T}\right) \boldsymbol{x}(t) \mathrm{d} t .
\end{gathered}
$$

From (27) to (29), the proof of Theorem 5 is completed.

Remark 6. It can be seen from Theorem 5 that the relationship between the adaptive control gain and the performance coefficient is obtained, and the guaranteed-performance cost related to the adaptive control gain is determined, which means that the guaranteed-performance cost can be regulated by changing the adaptive control gain. Then by the adaptive control strategy, the impacts of time-varying communication weights can be reduced in the adaptive guaranteed-performance consensus analysis. However, the performance function is not mentioned in [12-15]; let alone the guaranteed-performance cost. In this case, the consensus performance of multiagent systems cannot be guaranteed, which has less significance in practical applications. Furthermore, although time-varying communication weights is considered in $[13,14]$, there is no effective method to overcome its impacts; that is, the consensus analysis is more complicated and affected by time-varying communication weights.

(D) Convergence Speed Analysis. It should be noted that the Laplacian matrix $L$ related to the undirected graph $G$ is a semidefinite matrix. Then being the minimum nonzero eigenvalue of $L, \lambda_{2}$ is also said to be the algebraic connectivity satisfying

$$
\lambda_{2}=\min _{x \neq 0,1_{M}^{T} x=0} \frac{\boldsymbol{x}^{T}(t) L \boldsymbol{x}(t)}{\|\boldsymbol{x}(t)\|^{2}} .
$$

According to [23], one can see that in case there is no gain matrix in the consensus control protocol, the convergence 
speed is associated with the minimum nonzero eigenvalue $\lambda_{2}$. In other words, the larger the value of $\lambda_{2}$ is, the faster the convergence speed is.

For the sake of verifying the assumption that the proposed method can effectively regulate the convergence speed of multiagent system (1), we give the definition of the convergence coefficient as follows.

Definition 7. For multiagent system (1), the convergence coefficient $\rho(t)$ can be defined as

$$
\rho(t)=-\frac{\dot{V}(t)}{\widetilde{V}(t)} .
$$

where $V(t)$ is the Lyapunov function, $\dot{V}(t)$ is the time derivative of $V(t)$, and $\widetilde{V}(t)=\boldsymbol{\mu}^{T}(t) \boldsymbol{\mu}(t)$. Then the lower bound of $\rho(t)$ is shown as $\rho_{\min }$ which means the minimum convergence speed of multiagent system (1).

As a matter of fact, (29) originates from Definition 7. Then with regard to multiagent system (1) under the standard control protocol in [23], where $V(t)=\boldsymbol{x}^{T}(t) \boldsymbol{x}(t)$ and $\dot{V}(t)=$ $-2 \boldsymbol{x}^{T}(t) L \boldsymbol{x}(t)$, the convergence coefficient can be expressed as

$$
\rho(t)=-\frac{\dot{V}(t)}{\widetilde{V}(t)} \geq 2 \lambda_{2} .
$$

It can be seen that $\rho_{\min }=2 \lambda_{2}$; that is, the lower bound of $\rho(t)$ is directly associated with the algebraic connectivity. Since there exists the relationship between $\rho_{\min }$ and the algebraic connectivity, it is rational and convenient to use $\rho_{\min }$ to describe the convergence speed to some extent.

In the following, we determine the convergence coefficient of multiagent system (1) under control protocol (2) and compare it with the convergence coefficient under the standard consensus protocol. In order to ensure the effectiveness of this comparison, the control gain $\alpha$ is also considered in the standard consensus protocol as a reference, which means that $u_{k}(t)=\alpha \sum_{j \in N_{k}} w_{k j}(0)\left(x_{j}(t)-x_{k}(t)\right)$. In this case, one can directly obtain that $\rho_{\min , 1}=2 \alpha \lambda_{2}$.

Then substituting (18) into (20), the convergence coefficient of multiagent system (1) under control protocol (2) can be described as

$$
\rho(x)=-\frac{\dot{V}(x)}{\widetilde{V}(x)} \geq \alpha\left(2 \lambda_{2}+1\right) .
$$

Therefore, the following theorem can be obtained.

Theorem 8. The lower bound of the convergence coefficient of multiagent (1) under control protocol (2) is $\rho_{\min , 2}=\alpha\left(2 \lambda_{2}+1\right)$.

Remark 9. As the improvement of the convergence speed can save working time of multiagent systems in practice to some degree, it is significant to investigate how to improve the convergence speed when multiagent systems achieve consensus. Thus, the adjustable convergence speed is deduced, and the lower bound of the convergence coefficient under control protocol (2) is derived in this paper, which means

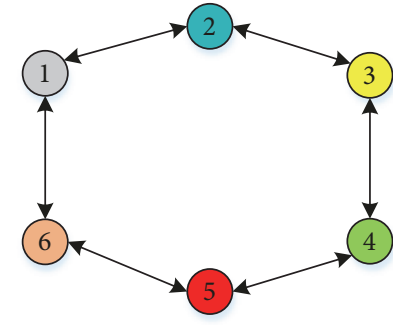

Figure 1: System topology.

that one can regulate the convergence speed by changing the adaptive control gain or the algebraic connectivity. However, in [12-15], the convergence speed is not considered; that is, one cannot regulate the consensus convergence according to the requirements in different environment.

Remark 10. By comparison, one can see that although changing the value of $\alpha$ can regulate the lower bound $\rho_{\min 1}$, the minimum nonzero eigenvalue of $L$ is usually small and cannot be determined precisely. Thus, it is also difficult to choose a proper control gain to adjust $\rho_{\min 1}$. On the contrary, there exists an extra item $\alpha$ in equation $\rho_{\min , 2}=\alpha\left(2 \lambda_{2}+1\right)$, which means that $\alpha$ can play a more important role in achieving the adjustable convergence speed. In other words, with the increase of the value of $\alpha, \rho_{\min , 2}$ is increased obviously and is less affected by the minimum nonzero eigenvalue of $L$. In this case, the convergence speed can be promoted more quickly. Furthermore, $\rho_{\min , 2}$ is linearly associated with $\alpha$ on account of fixed $\lambda_{2}$. As a result, the convergence speed of multiagent system (1) can be linearly regulated approximately. However, although many approaches have been used to adjust the convergence speed in [33-38]; all of them depend on the minimum nonzero eigenvalue of $L$ associated with the system topology.

\section{Simulation Results}

In this section, a simulation example is given to demonstrate the effectiveness of theoretical results shown in the previous analysis.

Consider a first-order multiagent system composed of six agents, where the system topology is depicted by the connected undirected graph shown in Figure 1. From the graph, one can get the Laplacian matrix:

$$
L=\left[\begin{array}{cccccc}
2 & -1 & 0 & 0 & 0 & -1 \\
-1 & 2 & -1 & 0 & 0 & 0 \\
0 & -1 & 2 & -1 & 0 & 0 \\
0 & 0 & -1 & 2 & -1 & 0 \\
0 & 0 & 0 & -1 & 2 & -1 \\
-1 & 0 & 0 & 0 & -1 & 2
\end{array}\right] .
$$

For adaptive guaranteed-performance control protocol (2), the adaptive control gain and the performance coefficient are chosen as $\alpha=1$ and $\zeta=0.4$, respectively. It can be seen 


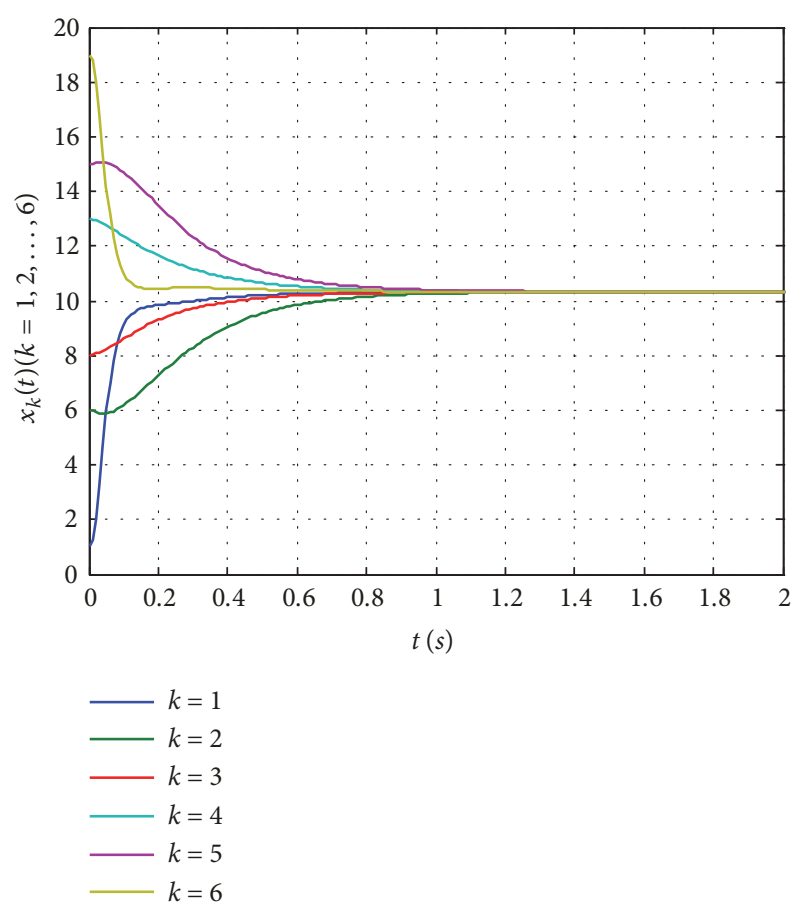

FIGURE 2: State trajectories under control protocol (2).

that these two parameters satisfy Theorem 5 . The initial state of each agent is $x(0)=[1,6,8,13,15,19]^{T}$.

Figure 2 shows the state trajectories of multiagent (1) with control protocol (2). One can see that the states of all agents reach a common value. Compared with the state trajectories of multiagent (1) under the standard consensus protocol in Figure 3, the convergence time of the former is much less; that is, the convergence speed is faster. Figure 4 depicts the guaranteed-performance function $J_{r}^{H}$ with $H=3$ and $J_{r}^{H}$ converges to a finite value with $J_{r}^{H}<J_{r}^{*}$. In Figure 5, it is shown that the relationship between the convergence time and $1 / \alpha$. One can obtain that the convergence time can be positively correlative approximately with $1 / \alpha$, provided that $\alpha$ is small enough. The simulation results illustrate that this multiagent system achieves guaranteed-performance consensus with an adjustable convergence speed.

\section{Discussions and Conclusions}

From Theorem 5, one can see that multiagent system (1) with control protocol (2) achieves adaptive guaranteedperformance consensus if the adaptive control gain $\alpha$ and the performance coefficient $\zeta$ satisfy certain numerical relationship, which can realize the trade-off design between consensus regulation performance and energy consumption. From Theorem 8, it can be obtained that the regulation performance of the adaptive control gain on the convergence speed can be much better if $\lambda_{2}$ far less than 1; that is, $\alpha$ can play a key role in the convergence speed regulation if the algebraic connectivity is very small. In this case, the

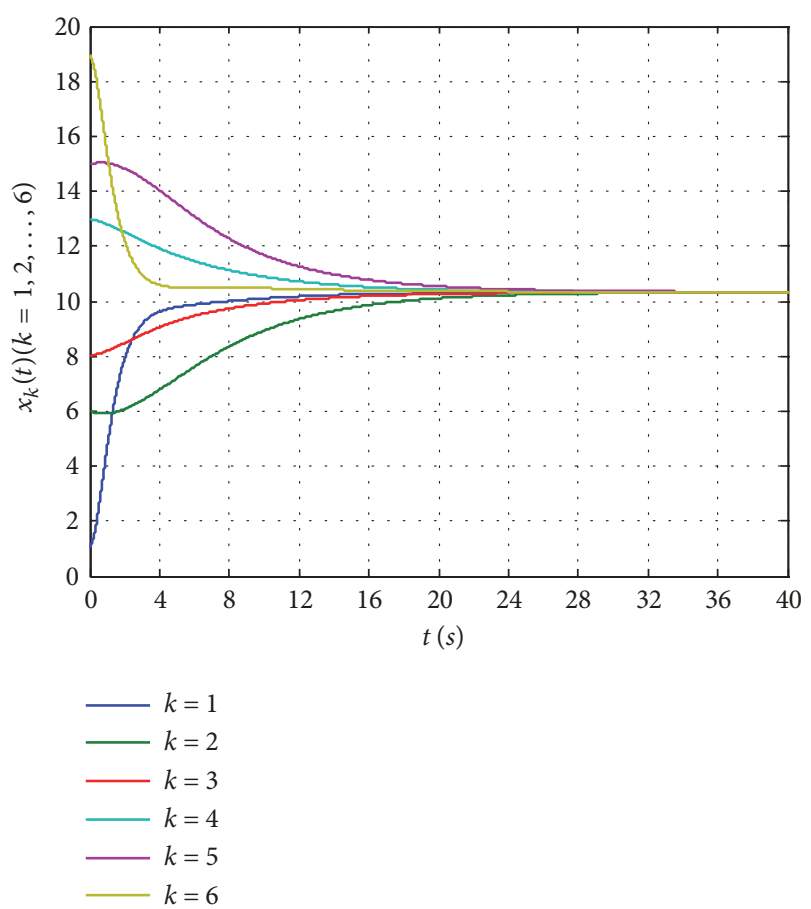

FIgURE 3: State trajectories under the standard consensus protocol.

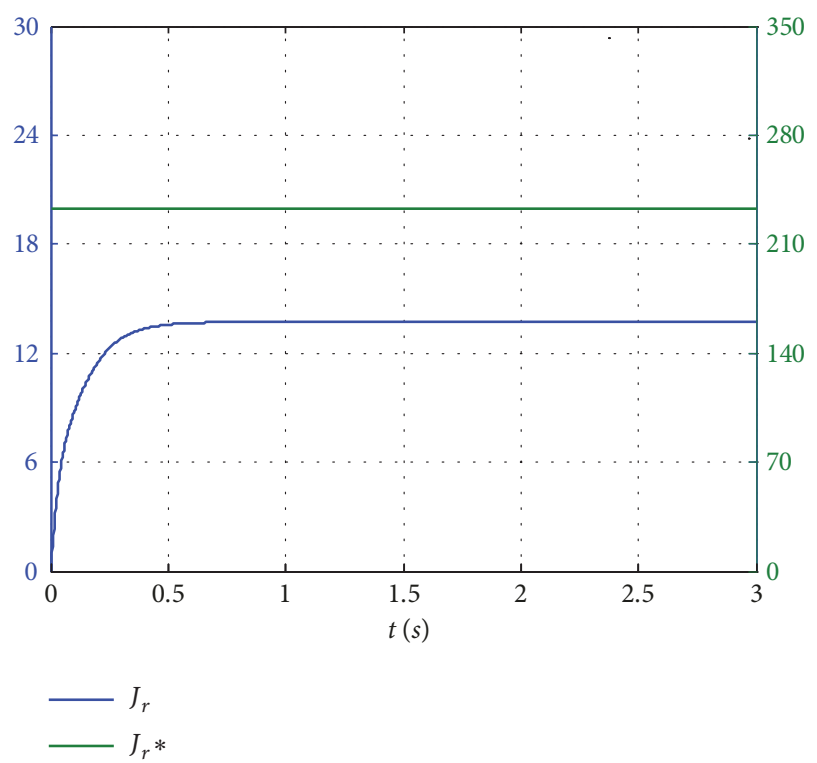

FIGURE 4: Guaranteed-performance function of multiagent system (1).

connected undirected system topology is simple, which can save the communication energy.

A new adaptive guaranteed-performance consensus scheme for multiagent systems with an adjustable convergence speed was proposed in this paper. The adaptive guaranteed-performance consensus protocol was presented by adjusting the communication weights among agents in the system topology. Sufficient conditions for adaptive guaranteed-performance consensus were obtained and the 


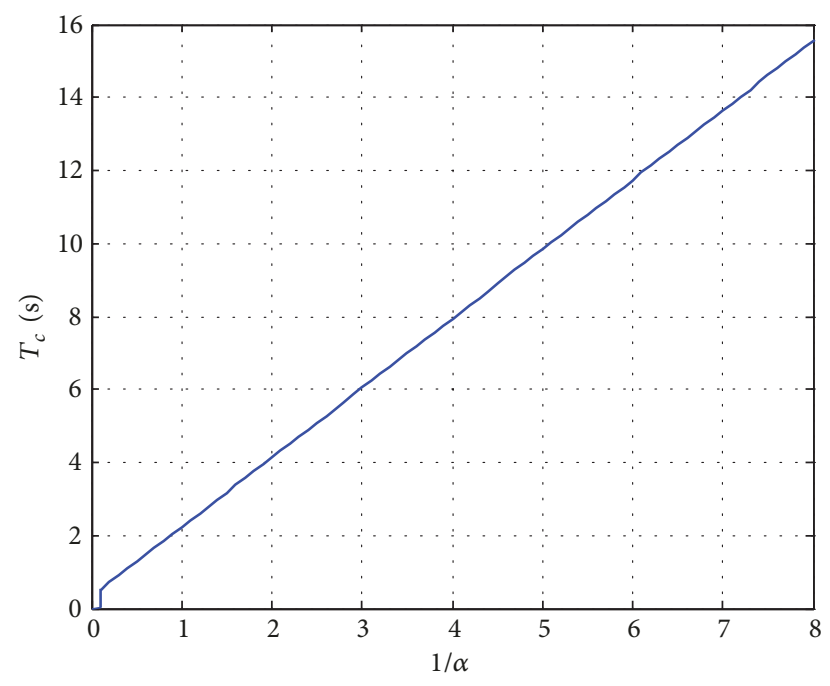

FIGURE 5: The relationship between convergence time and reciprocal of adaptive control gain.

guaranteed-performance cost was deduced. Then in order to indicate the convergence speed of multiagent systems, the convergence coefficient was defined, and it was proved that the convergence speed can be approximately linear adjustable by changing the adaptive control gain.

This paper assumes that the research objective is firstorder linear multiagent systems. The dynamics of each agent is described as the first-order integrators. In this case, the adaptive guaranteed-performance consensus analysis can be simplified by the specific structure of the multiagent system. However, due to the wide existence of nonlinear dynamics in practical systems, it is very meaningful to study nonlinear multiagent systems. Thus, we will focus on adaptive guaranteed-performance consensus problems for nonlinear multiagent systems by the Lipschitz condition in the future.

\section{Data Availability}

All the data in the simulation is included within this article.

\section{Conflicts of Interest}

The authors declare that there are no conflicts of interest regarding the publication of this paper.

\section{Acknowledgments}

I would like to express my faithful gratitude to all those who helped me during the writing of this paper. I gratefully acknowledge the help of my partners, Mr. Ning Cai and Mr. Jianxiang $\mathrm{Xi}$, who have offered me patient instruction in the academic studies. This work is supported by National Natural Science Foundation (NNSF) of China (Grants 61867005 and 61763040), by Fundamental Research Funds for the Central Universities (Grants 2019RC29 and 31920180115), by the Gansu Provincial First-Class Discipline Program of Northwest Minzu University (Grant 11080305), and by Research
Funds of SEAC China for the New Silk Road Economic Belt (Grant 2018-GMH-001).

\section{References}

[1] Y. Tang, H. Gao, W. Zou, and J. Kurths, "Distributed synchronization in networks of agent systems with nonlinearities and random switchings," IEEE Transactions on Systems, Man, and Cybernetics, Part B: Cybernetics, vol. 43, no. 1, pp. 358-370, 2013.

[2] N. Cai, M. He, Q. Wu, and M. J. Khan, "On almost controllability of dynamical complex networks with noises," Journal of Systems Science and Complexity, In press.

[3] H. Liu, T. Ma, F. L. Lewis, and Y. Wan, "Robust formation control for multiple quadrotors with nonlinearities and disturbances," IEEE Transactions on Cybernetics, In press.

[4] X. Dong, Y. Zhou, Z. Ren, and Y. Zhong, "Time-varying formation tracking for second-order multi-agent systems subjected to switching topologies with application to quadrotor formation flying," IEEE Transactions on Industrial Electronics, vol. 64, no. 6, pp. 5014-5024, 2017.

[5] T. Zheng, J. Xi, M. Yuan, and G. Liu, "Guaranteed-performance consensus design for Lipschitz nonlinear multiagent systems with jointly connected topologies," International Journal of Robust and Nonlinear Control, vol. 29, no. 11, pp. 3627-3649, 2019.

[6] Z.-Y. Tan, N. Cai, J. Zhou, and S.-G. Zhang, "On performance of peer review for academic journals: analysis based on distributed parallel system," IEEE Access, vol. 7, no. 99, pp. 19024-19032, 2019.

[7] X. Yang, W. Yu, R. Wang, G. Zhang, and F. Nie, "Fast spectral clustering learning with hierarchical bipartite graph for largescale data," Pattern Recognition Letters, In press.

[8] P. Lin, Y. Wang, H. Qi, and Y. Hong, "Distributed consensusbased K-means algorithm in switching multi-agent networks," Journal of Systems Science and Complexity, vol. 31, no. 5, pp. 1128-1145, 2018.

[9] J. Qu, Z. Ji, C. Lin, and H. Yu, "Fast consensus seeking on networks with antagonistic interactions," Complexity, vol. 2018, Article ID 7831317, 15 pages, 2018.

[10] Y. Zhang, J. Sun, H. Liang, and H. Li, "Event-triggered adaptive tracking control for multiagent systems with unknown disturbances," IEEE Transactions on Cybernetics, pp. 1-12, 2018.

[11] Y. Zhang, H. Li, J. Sun, and W. He, "Cooperative adaptive eventtriggered control for multiagent systems with actuator failures," IEEE Transactions on Systems, Man, and Cybernetics: Systems, pp. 1-10, 2018.

[12] H. Kim, H. Shim, and J. H. Seo, "Output consensus of heterogeneous uncertain linear multi-agent systems," IEEE Transactions on Automatic Control, vol. 56, no. 1, pp. 200-206, 2011.

[13] X. Lin and Y. S. Zheng, "Finite-time consensus of switched multi-agent systems," IEEE Transactions on Systems, Man, and Cybernetics: Systems, vol. 47, no. 7, pp. 1535-1545, 2017.

[14] M. J. Park, O. M. Kwon, J. H. Park, S. Lee, and E. Cha, "A new analysis on leader-following consensus for switched multiagent systems with time-varying probabilistic self-delays," International Journal of Control, Automation, and Systems, vol. 13, no. 3, pp. 611-619, 2015.

[15] J. Xi, M. He, H. Liu, and J. Zheng, "Admissible output consensualization control for singular multi-agent systems with time delays," Journal of The Franklin Institute, vol. 353, no. 16, pp. 4074-4090, 2016. 
[16] B. Hu, Z. Guan, X. Jiang, M. Chi, and L. Yu, "On consensus performance of nonlinear multi-agent systems with hybrid control," Journal of The Franklin Institute, vol. 353, no. 13, pp. 3133-3150, 2016.

[17] Y. Cao and W. Ren, "Optimal linear-consensus algorithms: an lqr perspective," IEEE Transactions on Systems, Man, and Cybernetics, Part B: Cybernetics, vol. 40, no. 3, pp. 819-830, 2010.

[18] Z.-H. Guan, B. Hu, M. Chi, D.-X. He, and X.-M. Cheng, "Guaranteed performance consensus in second-order multiagent systems with hybrid impulsive control," Automatica, vol. 50, no. 9, pp. 2415-2418, 2014.

[19] J. Xi, C. Wang, H. Liu, and L. Wang, "Completely distributed guaranteed-performance consensualization for highorder multiagent systems with switching topologies," IEEE Transactions on Systems, Man, and Cybernetics: Systems, vol. 49, no. 7, pp. 1338-1348, 2019.

[20] J. Xi, C. Wang, H. Liu, and Z. Wang, "Dynamic output feedback guaranteed-cost synchronization for multiagent networks with given cost budgets," IEEE Access, vol. 6, pp. 28923-28935, 2018.

[21] J. Xu, G. Zhang, J. Zeng, J. Xi, and B. Du, "Robust guaranteed cost consensus for high-order discrete-time multi-agent systems with parameter uncertainties and time-varying delays," IET Control Theory \& Applications, vol. 11, no. 5, pp. 647-667, 2017.

[22] J. Xi, Z. Fan, H. Liu, and T. Zheng, "Guaranteed-cost consensus for multiagent networks with Lipschitz nonlinear dynamics and switching topologies," International Journal of Robust and Nonlinear Control, vol. 28, no. 7, pp. 2841-2852, 2018.

[23] R. Olfati-Saber and R. M. Murray, "Consensus problems in networks of agents with switching topology and time-delays," IEEE Transactions on Automatic Control, vol. 49, no. 9, pp. 15201533, 2004.

[24] R. Olfati-Saber, J. A. Fax, and R. M. Murray, "Consensus and cooperation in networked multi-agent systems," Proceedings of the IEEE, vol. 95, no. 1, pp. 215-233, 2007.

[25] M. Fiedler, "Algebraic connectivity of graphs," Czechoslovak Mathematical Journal, vol. 98, no. 23, pp. 298-305, 1973.

[26] R. Olfati-Saber, "Ultra-fast consensus in small-world networks," in Proceedings of the American Control Conference (ACC '05), pp. 2371-2378, IEEE, Portland, Ore, USA, June 2005.

[27] Y. Kim and M. Mesbahi, "On maximizing the second smallest eigenvalue of a state-dependent graph laplacian," IEEE Transactions on Automatic Control, vol. 51, no. 1, pp. 116-120, 2006.

[28] M. Paulus and L. Kristoufek, "Worldwide clustering of the corruption perception," Physica A: Statistical Mechanics and its Applications, vol. 428, pp. 351-358, 2015.

[29] Z. Zhou, S. Lin, and Y. Xi, "A fast network partition method for large-scale urban traffic networks," Journal of Control Theory and Applications, vol. 11, no. 3, pp. 359-366, 2013.

[30] Y. Xi and X. Li, "Hierarchical structure design for multi-agent consensus," Control Theory \& Applications, vol. 32, no. 9, pp. 1191-1199, 2015.

[31] M. Epstein, K. Lynch, K. H. Johansson, and R. M. Murray, "Using hierarchical decomposition to speed up average consensus," International Federation of Automatic Control World Congress, pp. 612-618, 2008.

[32] M. Epstein, Managing Information in Networked and MultiAgent Control Systems, American California Institute of Technology, 2007.
[33] D. Yuan, S. Xu, H. Zhao, and Y. Chu, "Accelerating distributed average consensus by exploring the information of secondorder neighbors," Physics Letters A, vol. 374, no. 24, pp. 24382445, 2010.

[34] H. Pan, X. Nian, and L. Guo, "Second-order consensus in multiagent systems based on second-order neighbours' information," International Journal of Systems Science, vol. 45, no. 5, pp. 902914, 2014

[35] H. Peng, G. Qi, and A. Sheng, "Pseudo multi-hop relay distributed consensus algorithm," Control Theory \& Applications, vol. 5, no. 29, pp. 623-628, 2012.

[36] H. Peng, A. Sheng, and W. Wang, "Pseudo multi-hop distributed consensus algorithm under directed topologies," in Proceedings of the 2012 12th International Conference on Control, Automation, Robotics and Vision, ICARCV 2012, pp. 1616-1619, China, December 2012.

[37] H. Zhang, M. Z. Q. Chen, and G. Stan, "Fast consensus via predictive pinning control," IEEE Transactions on Circuits and Systems I: Regular Papers, vol. 58, no. 9, pp. 2247-2258, 2011.

[38] T. C. Aysal, B. N. Oreshkin, and M. J. Coates, "Accelerated distributed average consensus via localized node state prediction," IEEE Transactions on Signal Processing, vol. 57, no. 4, pp. 1563 1576, 2009.

[39] Z. Q. Zhang and J. D. Cao, "Periodic solutions for complexvalued neural networks of neutral type by combining graph theory with coincidence degree theory," Advances in Difference Equations, vol. 2018, no. 261, 2018.

[40] Z. Li, Z. Duan, G. Chen, and L. Huang, "Consensus of multiagent systems and synchronization of complex networks: a unified viewpoint," IEEE Transactions on Circuits and Systems I: Regular Papers, vol. 57, no. 1, pp. 213-224, 2009.

[41] Z. Zhang and L. Ren, "New sufficient conditions on global asymptotic synchronization of inertial delayed neural networks by using integrating inequality techniques," Nonlinear Dynamics, vol. 95, no. 2, pp. 905-917, 2019. 


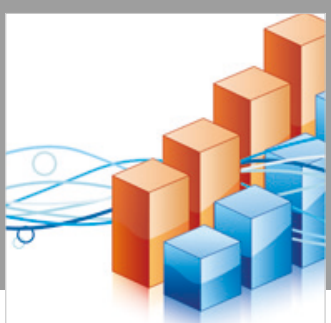

Advances in

Operations Research

\section{-n-m}
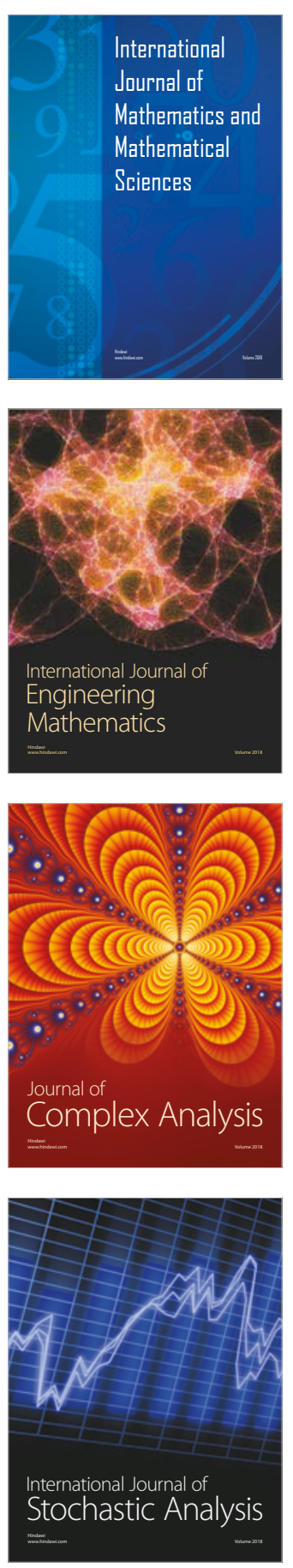
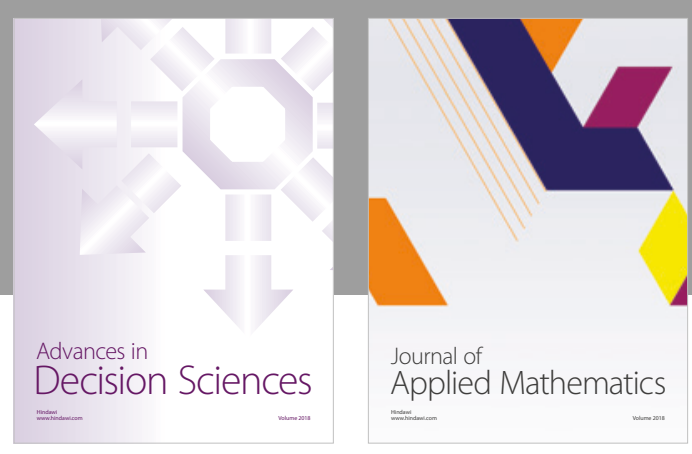

Journal of

Applied Mathematics
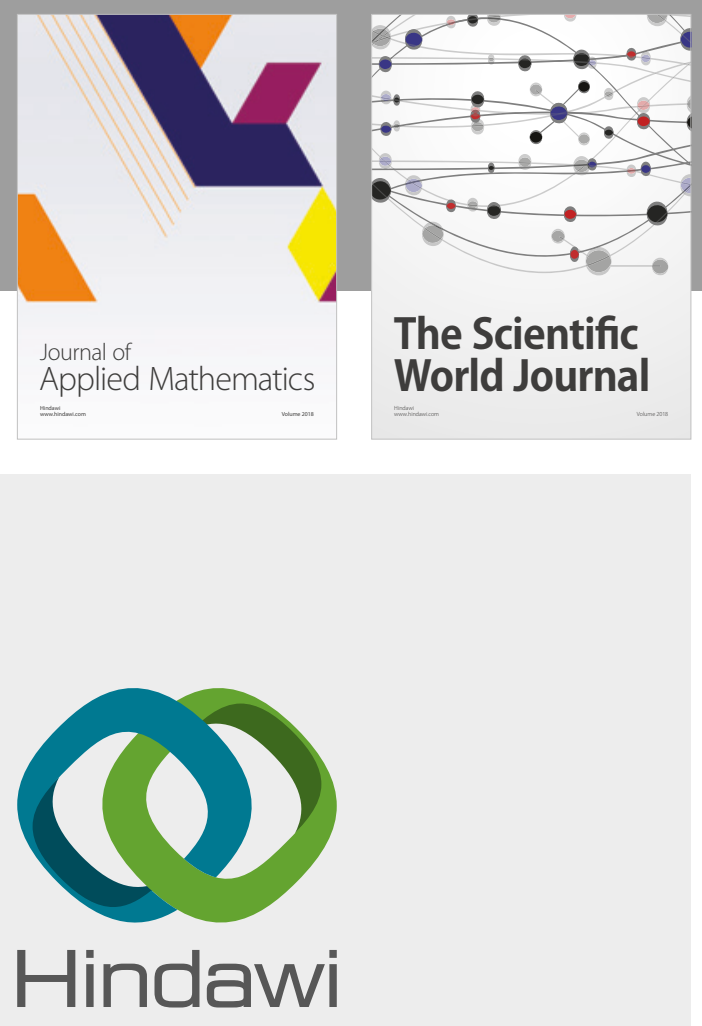

Submit your manuscripts at

www.hindawi.com

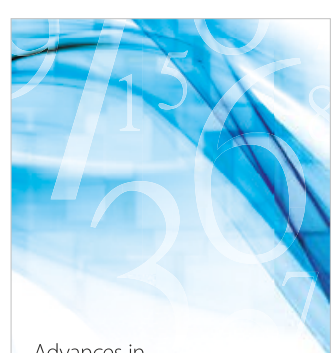

Advances in
Numerical Analysis
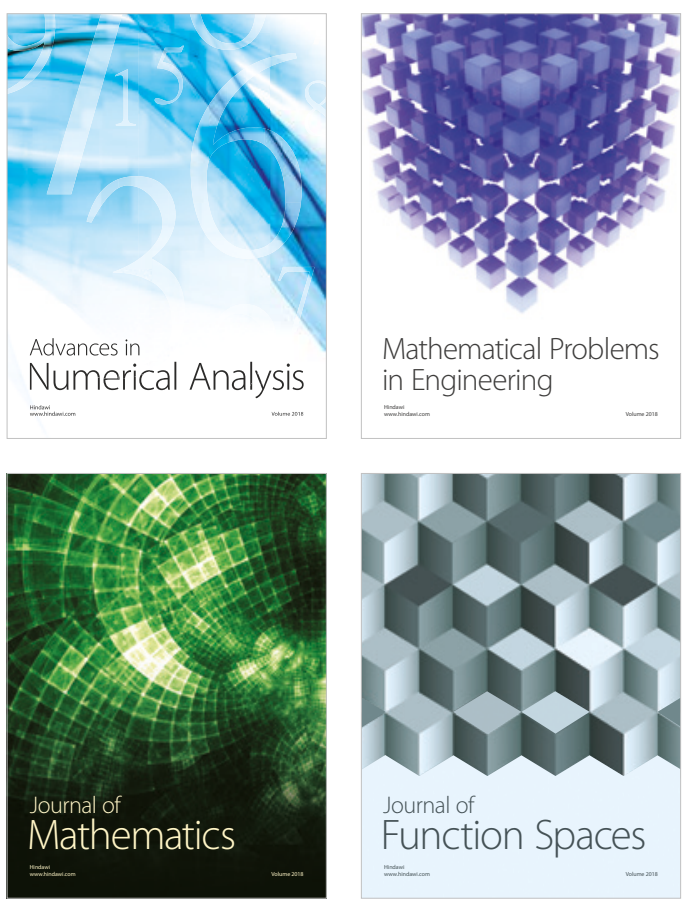

Mathematical Problems in Engineering

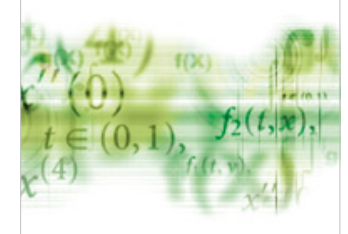

International Journal of

Differential Equations

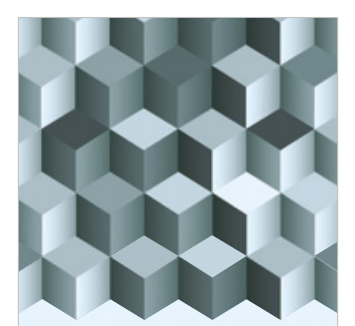

Journal of

Function Spaces
The Scientific

World Journal

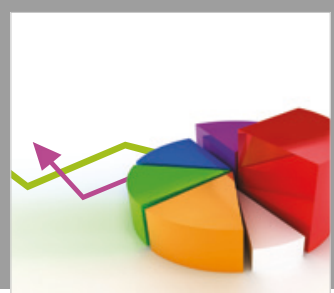

Journal of

Probability and Statistics
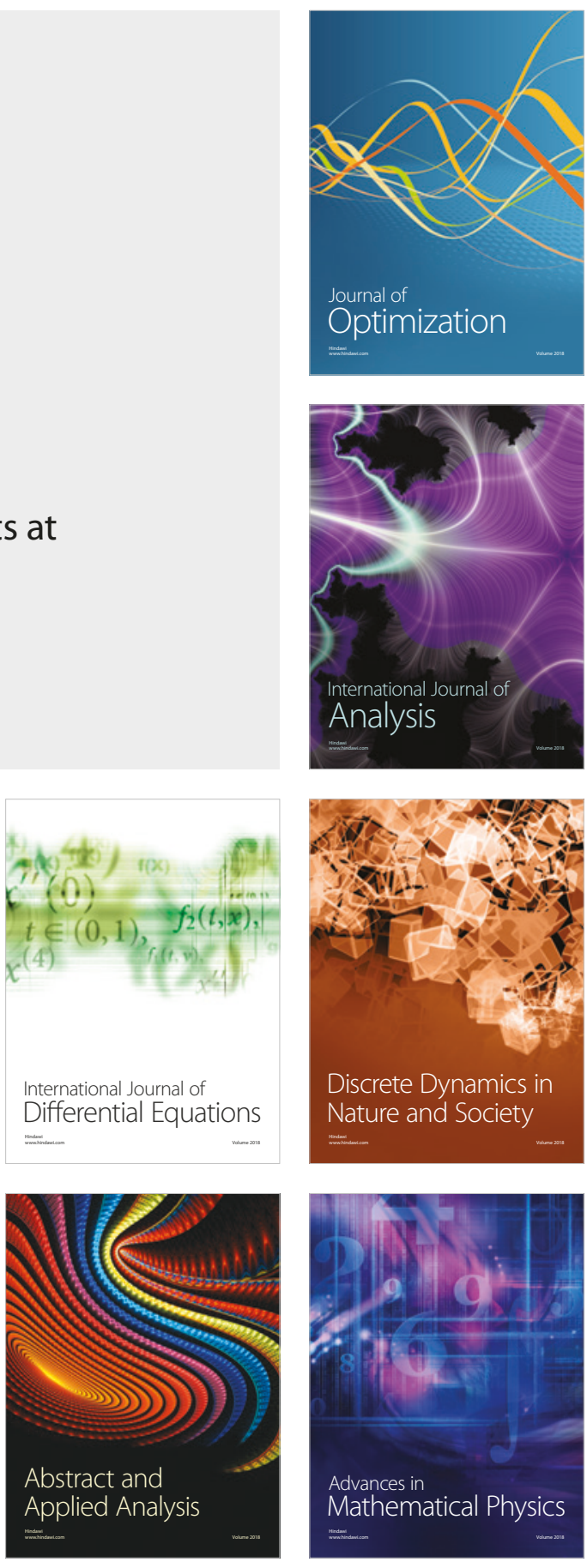